" $A C X D Y B$ is a hexagon inscribed in a line-pair", or " $C X$ and $B Y$ form homographic pencils with a common ray and two pairs of corresponding rays which meet at infinity " Or, as analytical methods would not be forbidden by the regulations, they might resort to areal coordinates.

$$
\text { Yours, etc., }
$$

A. Robson.

SIR,-Scholarship candidates given Mr Newling's 1894 Tripos question solved it by means of similarity, overlooking the construction which gave his neat proof by congruence.

I suggest that the examiners expected the following (longer) solution. I use Mr. Newling's notation, but produce $B Y$ to cut $A C$ at $P$ and draw $A Z$ parallel to $B Y$ cutting $B C$ at $Z$; also $A X Y$ cuts $B C$ at $O$. Then

$$
(A X O Y)=D(A X O Y)=D(A E C Y)=-1,
$$

for $A E=E C$ and $D Y$ is parallel to $A C$;

$$
(Z C O B)=A(Z C O B)=A(Z P Y B)=-1,
$$

for $B Y=Y P$ and $A Z$ is parallel to $B Y$. From these it follows that $C X$ is parallel to $A Z$ and $B Y$

$$
\text { Yours, etc., }
$$

H. V Styler.

\title{
PARTIAL FRACTIONS.
}

To the Editor of the Mathematical Gazette.

SIR,-Teachers may be interested in points which arise from time to time in examination answers, as a guide to possible misunderstanding by their pupils. I have recently found a rather large number of candidates who attempted to evaluate an integral of the form

$$
\int \frac{d x}{\left(a+b x^{2}\right) \sqrt{ }\left(c+d x^{2}\right)}
$$

by the step

$$
\frac{1}{\left(a+b x^{2}\right) \sqrt{ }\left(c+d x^{2}\right)} \equiv \frac{A x+B}{a+b x^{2}}+\frac{C}{\sqrt{ }\left(c+d x^{2}\right)}
$$

with variants of the actual form of "partial fractions" By various devices the coefficients $A, B, C$ were calculated.

I am writing because it seems to me that such work implies a fundamental misunderstanding of partial fractions themselves. The mechanical calculations are effected (a conference on really tidy methods would help examiners-and candidates!) but it is possible that many candidates do not fully understand just why their steps are legitimate.

Of course it is possible to perform mathematical calculations without fully understanding all the theory, as, say, in logarithms. But here positive dangers arise, and a treatment of the subject which excludes these seems desirable.

Yours, etc.,

E. A. Maxwell.

Queens' College, Cambridge.

\section{STARRED QUESTIONS.}

\section{To the Editor of the Mathematical Gazette.}

SIR,-The question of what makes a good scholarship question is an interesting one on which a great variety of opinion must be held by your readers. It would be valuable to have views from university teachers as well as school teachers. 